\title{
Cyclosporin A treatment of young children with newly-diagnosed Type 1 (insulin-dependent) diabetes mellitus
}

\author{
M.Jenner ${ }^{1}$, G. Bradish ${ }^{1}$, C. Stiller ${ }^{2}$, P. Atkison ${ }^{1,2}$ for the London Diabetes Study Group \\ ${ }^{1}$ Department of Paediatrics, Children's Hospital of Western Ontario, University of Western Ontario, and ${ }^{2}$ Multi-Organ Transplant Service, \\ University Hospital, London, Ontario, Canada
}

\begin{abstract}
Summary. Several studies have demonstrated the efficacy of cyclosporin A in modifying the initial course of Type 1 (insulin-dependent) diabetes mellitus in older children and adults but none have reported the effects in very young children. We treated 14 newly-diagnosed Type 1 diabetic patients aged 22 months to 95 months with cyclosporin A. Mean insulin dose at entry was $0.7 \pm 0.07 \mathrm{IU} \cdot \mathrm{kg}^{-1} \cdot \mathrm{day}^{-1}$. Initial cyclosporin A dose was $10 \mathrm{mg} \cdot \mathrm{kg}^{-1} \cdot \mathrm{day}^{-1}$. Insulin dose reached a nadir of $0.13 \mathrm{IU} \cdot \mathrm{kg}^{-1}$. day ${ }^{-1}$ by 180 days. Mean glucagon-stimulated connecting peptide levels were maximal at 6 months $(0.75 \mathrm{nmol} / \mathrm{l})$ and were maintained while on cyclosporin A. Insulin was discontinued in four patients for $4,12,15$ and 30 months respectively. In five other patients the insulin dose was less than $0.15 \mathrm{IU} \cdot \mathrm{kg}^{-1} \cdot \mathrm{day}^{-1}$ for at least 3 months. Glycated haemoglobin levels for all patients were within the normal range. Side effects included anorexia, stomach pains, poor weight gain, hypertrichosis, gum hyperplasia, mild anaemia and elevated creatinine. All patients have now dis-
\end{abstract}

continued cyclosporin A and all but one have been followed for 5 years after discontinuation. Reasons for discontinuing cyclosporin A included exposure to chicken pox (varicella), non-resolving otitis media, incomplete or no response and relapse. All side effects have resolved since the treatment was discontinued. Following discontinuation of cyclosporin $\mathrm{A}$ insulin requirements and glycated hemoglobin levels increased while glucagon-stimulated connecting peptide levels declined dramatically. In summary, a small number of very young patients treated with cyclosporin A achieved noninsulin requiring remissions while partial remissions occurred in several other patients and endogenous insulin production was maintained. Side effects to the drug occurred although there have been no long-term consequences.

Key words: Cyclosporin A, Type 1 (insulin-dependent) diabetes mellitus, children, side effects.
A number of studies, including two randomized - double blind placebo controlled trials $[1,2]$ have now demonstrated the efficacy of cyclosporin A (CsA) in modifying the initial course of Type 1 (insulin-dependent) diabetes mellitus. Three recent reports [3-5] have suggested that CsA may be particularly useful in treating young, newlydiagnosed patients between the ages of 7-15 years since they show higher rates of non-insulin requiring remissions. Significant side effects, particularly nephrotoxicity, have limited the use of CsA in these patients. However, nephrotoxicity appears to be dose related and does not occur, or is reversible, at lower doses [6].

This pilot study was initiated in 1983 following the encouraging results of our early studies of CsA treatment of newly-diagnosed adults with Type 1 diabetes [7]. We wanted to assess the potential benefits for young children as extreme variability in activity and diet make conventional diabetes management difficult in this age group. In addition very young children have only small amounts of subcutaneous fat which often restrict the number of injec- tion sites. CsA therapy was successful in some of these younger patients aged 22 months to 95 months. However, difficulties with administration of CsA, its side effects and variable results, resulted in discontinuation of CsA therapy in all of these patients. With the exception of one patient these children have now been followed-up for a minimum of 5 years after discontinuation of CsA. This report summarizes the results of CsA therapy in this age group and their long-term follow-up.

\section{Subjects and methods}

Approval for this study was obtained from the Human Ethics Committee at the University of Western Ontario. Patients ranged in age from 22 months to 95 months. Diagnosis of Type 1 diabetes was established by conventional means including classic symptoms in association with gross elevations of blood glucose. Exclusion criteria included: a history of hepatic, renal or bone marrow disease, a history of malignancy, evidence of Epstein-Barr virus, cytomegalovirus or other uncontrolled infections or other contraindications to immuno- 


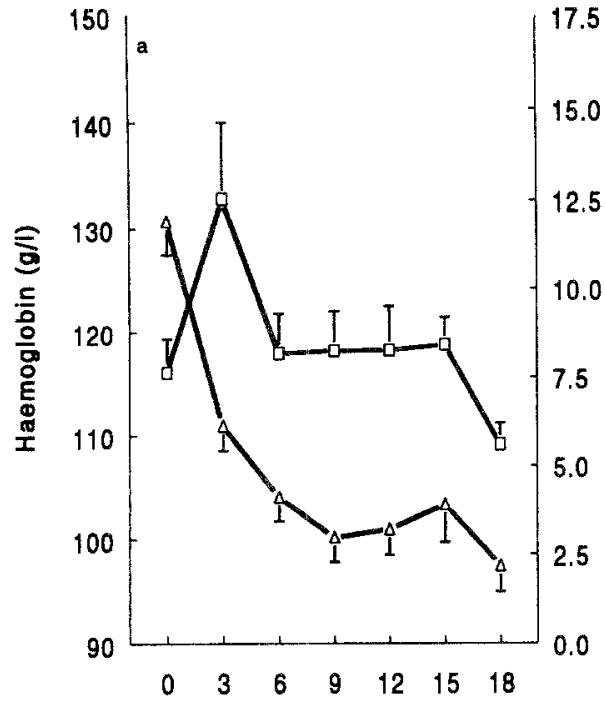

Cyclosporin A therapy (months)

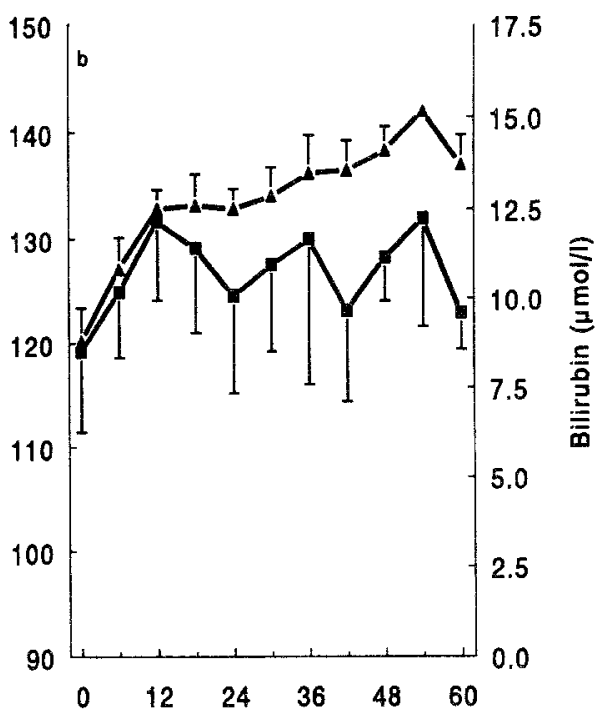

Post-discontinuation of therapy (months)
Fig. 1a,b. Effects of cyclosporin A (CsA) therapy on haemoglobin levels (triangles) and bilirubin (squares). Values are shown as mean ( \pm SEM) post-entry to study while on CsA therapy (a, open symbols) and after discontinuation of CsA (b, closed symbols). The $n$ for each time point varied slightly and for haemoglobin was: $13,12,10$, $9,5,5,2$ and for bilirubin: $10,9,10,9,5$, 5,2 at $0,3,6,9,12,15,18$ months respectively post-entry while on CsA. Following discontinuation of CsA the $n: 10$, $12,11,9,9,10,9,6,8,3,6$, for haemoglobin and $8,10,10,9,9,8,9,6,7,4,5$, for bilirubin at $0,6,12,18,24,30,36,42$, 48,54 , and 60 months respectively suppression. All but two of the patients were entered into the study within 6 weeks of diagnosis of diabetes. The remaining two patients were started within 7 and 11 weeks following diagnosis. Patients were entered into this study on a case-by-case basis following discussion of potential side effects of CsA therapy with the families and after obtaining informed consent. The goals of therapy were similar to those previously reported for the Canadian European randomized control trial [2]. In brief, we attempted to keep before meal and before bedtime glucose levels less than $7.8 \mathrm{mmol} / 1$ and glycated haemoglobin levels within the normal range.

At the start of therapy patients were admitted to hospital for stabilization of blood glucose. Cyclosporin A treatment was initiated while in hospital at a dose of $10 \mathrm{mg} \cdot \mathrm{kg}^{-1} \cdot \mathrm{day}^{-1}$ in two divided oral doses. The CsA dose was adjusted to maintain serum trough levels between $150-350 \mathrm{ng} / \mathrm{ml}$ as determined by a polyclonal radioimmunoassay. The antibody was kindly provided by Sandoz (Basel, Switzerland). Blood was obtained at the start of the study for baseline measurements. Patients were seen at frequent intervals following diagnosis at which time laboratory studies were repeated, blood pressure, height and weight were recorded. Insulin adjustments were made to minimize the dose of insulin while maintaining target glycaemic control. Endogenous insulin secretion was assessed every 3 months with $1 \mathrm{mg}$ glucagon administered i.v, following an overnight fast. Blood samples were collected for connecting peptide levels at 0 and $6 \mathrm{~min}$ following injection.

Patients were considered to be in remission if they maintained target glycaemia without the need for exogenous insulin or partial remission if they required less than $0.15 \mathrm{IU} \cdot \mathrm{kg}^{-1} \cdot$ day $^{-1}$ s.c. for at least 2 weeks. A diagnosis of relapse was made if there was deterioration in glycaemic parameters or a necessary increase in insulin requirements beyond $0.15 \mathrm{IU} \cdot \mathrm{kg}^{-1} \cdot$ day $^{-1} \mathrm{~s}$.c. for more than 4 weeks in order to maintain target glycaemic control or both in which case cyclosporin A was then discontinued. Patients who did not achieve insulin doses of less than $0.15 \mathrm{IU} \cdot \mathrm{kg}^{-1} \cdot \mathrm{day}^{-1}$ were considered to be non-responsive.

\section{Results}

Fourteen patients (four male, ten female) entered the study ranging in age from 22 months to 95 months. The mean age at entry was $5.4 \pm 0.6$ years. With the exception of two patients, all others were enrolled in the study within 6 weeks of diagnosis and the remaining two were entered at 7 and 11 weeks following diagnosis. The mean duration from diagnosis to initiation of $\mathrm{CsA}$ in the 12 patients was $11.3 \pm 2$ days. Ninety-three percent of the patients were HLA DR3 positive, DR4 positive or both. One patient was discontinued from the study 7 days after initiating CsA therapy due to exposure to chicken pox (varicella), this patient was not included in the data analysis. The remaining 13 patients were continued on CsA therapy for varying lengths of time. CsA was discontinued for a variety of reasons, one patient, was discontinued due to exposure to chicken pox at 10 days. Three patients discontinued $\mathrm{CsA}$ therapy at 3,8 and 9 months respectively, due to non-resolving otitis media. Six patients discontinued CsA at 3, 4, 6, 10 (two patients) and 16 months respectively, due to an inability to discontinue exogenous insulin although five of these had insulin requirements of less than $0.15 \mathrm{IU}$. $\mathrm{kg}^{-1} \cdot \mathrm{day}^{-1}$ for more than 3 months. Four patients who had undergone a non-insulin requiring remission, discontinued CsA therapy at 9,16,22 and 36 months respectively, due to their loss of a non-insulin-requiring state. There were no obvious differences in the achieved degree of glycaemic control during the initial (10 to 14 days) treatment period with CsA to account for the response to CsA. Of the four patients who discontinued insulin three were female. All started CsA within 4 weeks of diagnosis. Their ages at the start of CsA therapy were 39,77,78 and 95 months. Thus, they tended to be older and had a short duration of diabetes. Neither patient who started CsA therapy beyond 6 weeks after diagnosis achieved even a partial remission (less than $0.15 \mathrm{IU} \cdot \mathrm{kg}^{-1} \cdot \mathrm{day}^{-1}$ of insulin). Of the 13 patients on CsA five were lost to follow-up at various times after discontinuing $\mathrm{CsA}$.

\section{Side effects of CsA therapy}

Both minor and major side effects to CsA therapy were seen in these patients. Minor side effects included gingival hyperplasia (most patients), hypertrichosis (most patients), anorexia (occasional) and abdominal pains (occa- 


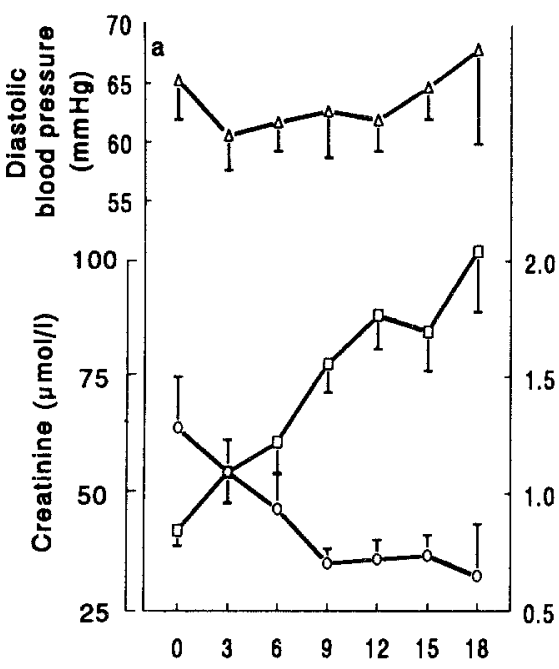

Cyclosporin A therapy (months)

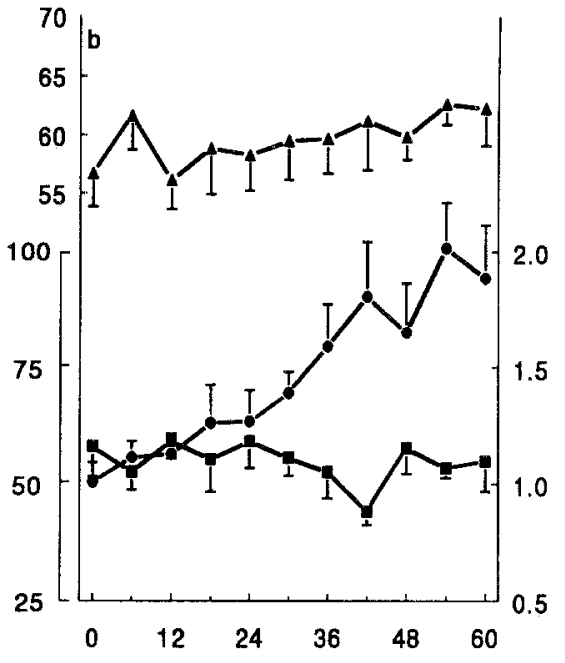

Post-discontinuation of therapy (months)
Fig. 2a,b. Effects of cyclosporin A (CsA) on diastolic blood pressure (triangles), creatinine (squares) and creatinine clearance (circles). Values are shown as the mean $( \pm$ SEM) by the open symbols post-entry into study while on CsA therapy (a) and following discontinuation of CsA therapy by the closed symbols (b). The $n$ for each time point varied slightly and for diastolic blood pressure was: 7,8 , $9,8,5,5,2$ while creatinine was: 13,12 , $10,9,5,5,2$ and creatinine clearance was: $10,12,10,9,5,5,2$, at $0,3,6,9,12,15$ and 18 months respectively post-entry while on CsA. Following discontinuation of CsA: the $n$ for diastolic blood pressure was: $8,12,11,9,6,9,7,8,7,3,7$, for creatinine: $9,13,12,8,9,9,8,6,8,4,7$ and for creatinine clearance: $10,11,11,9$, $8,10,8,6,8,4,7$, at $0,6,12,18,24,30,36$, $42,48,54$ and 60 months respectively sional). As shown in Figure 1, haemoglobin decreased on CsA therapy. Haemoglobin decreased from a mean of $130.8 \pm 11.8 \mathrm{~g} / \mathrm{l}$ at the start of CsA therapy to $97.5 \pm 3.5 \mathrm{~g} / \mathrm{l}$ at 18 months. The decline in haemoglobin was not serious enough to discontinue CsA. There was little, if any, effect on serum bilirubin measurements. As shown in Figure 2, diastolic blood pressure tended to increase on CsA therapy although there was considerable variability. Serum creatinine levels increased on CsA from a mean of $42.1 \pm 3.3 \mu \mathrm{mol} / 1$ to a high of $102 \pm 13.0 \mu \mathrm{mol} / 1$ at 18 months in the remaining two patients. Calculated creatinine clearance (Cockroft-Gault formula) declined in patients on CsA from a mean value at the start of therapy of $1.28 \pm 0.69 \mathrm{ml} / \mathrm{s}$ to a nadir of $0.65 \pm 0.31 \mathrm{ml} / \mathrm{s}$ at 18 months.

Growth was generally unaffected by CsA therapy in both male and female patients with a minor decrease in the linear growth of one male and one female patient. This was not considered significant and CsA was not discontinued. In addition, diminished appetite occurred in some patients, and close dietary supervision was necessary to maintain adequate caloric intake.

\section{Metabolic effecs of CsA therapy}

Non-insulin requiring remissions were achieved in four patients, who at the start of therapy were 39, 77, 94 and 78 months of age. The duration of the non-insulin requiring remissions were $4,12,15$ and 30 months, respectively. The one patient who remained insulin-free for 1 year had a brief ( 2 week) relapse at 6 months followed by a second non-insulin requiring remission which lasted 4 months before another relapse occurred. In an additional five patients, the insulin requirements declined to less than $0.15 \mathrm{IU} \cdot \mathrm{kg}^{-1} \cdot \mathrm{day}^{-1}$ for more than 3 months.

As shown in Figure 3 the mean dose of insulin declined to a low at 6 months of $0.13 \mathrm{IU} \cdot \mathrm{kg}^{-1} \cdot \mathrm{day}^{-1}$. The two patients still on CsA therapy at 18 months did not require insulin. The mean values of glucagon-stimulated connecting peptide (GSCP; Fig.3) were maximal at 6 months $(0.75 \mathrm{nmol} / \mathrm{l})$ and remained stable until discontinuation of
CsA. Finally, glycated haemoglobin levels declined to a minimum of $8.9 \%$ at 3 months and then remained constant while on CsA therapy. The normal range for the colorimetric glycated haemoglobin in our laboratory was $7.8 \pm 0.92 \%$.

\section{Side effects of post-discontinuation of $C s A$}

All CsA-related side effects resolved shortly after discontinuing CsA. These included the cosmetic (gingival hyperplasia, hypertrichosis) as well as other minor side effects such as abdominal cramps and decreased appetite. More significant side effects noted during CsA therapy improved following discontinuation. Haemoglobin progressively increased and returned to normal values while bilirubin concentrations did not show any significant change (Fig. 1). Diastolic blood pressure decreased slightly following discontinuation of CsA therapy while creatinine decreased and creatinine clearance increased (Fig. 2). Growth has been normal (data not shown).

Metabolic parameters post-discontinuation of CsA (Fig. 3)

Insulin requirements increased steadily following discontinuation of CsA therapy. This was accompanied by a decline in GSCP levels. By 24 months following discontinuation there was no measurable GSCP in any patient. Glycated haemoglobin levels increased to a maximum of $14.5 \%, 54$ months following discontinuation of CsA therapy.

\section{Discussion}

Stiller and colleagues [8] initially suggested a possible therapeutic role for CsA therapy in treating individuals with Type 1 diabetes. Their initial studies on diabetesprone $\mathrm{BB} / \mathrm{W}$ rats indicated that CsA therapy could prevent diabetes and insulitis in these animals. Subsequently, they reported that CsA therapy in newly-diagnosed individuals with Type 1 diabetes resulted in non-insulin re- 


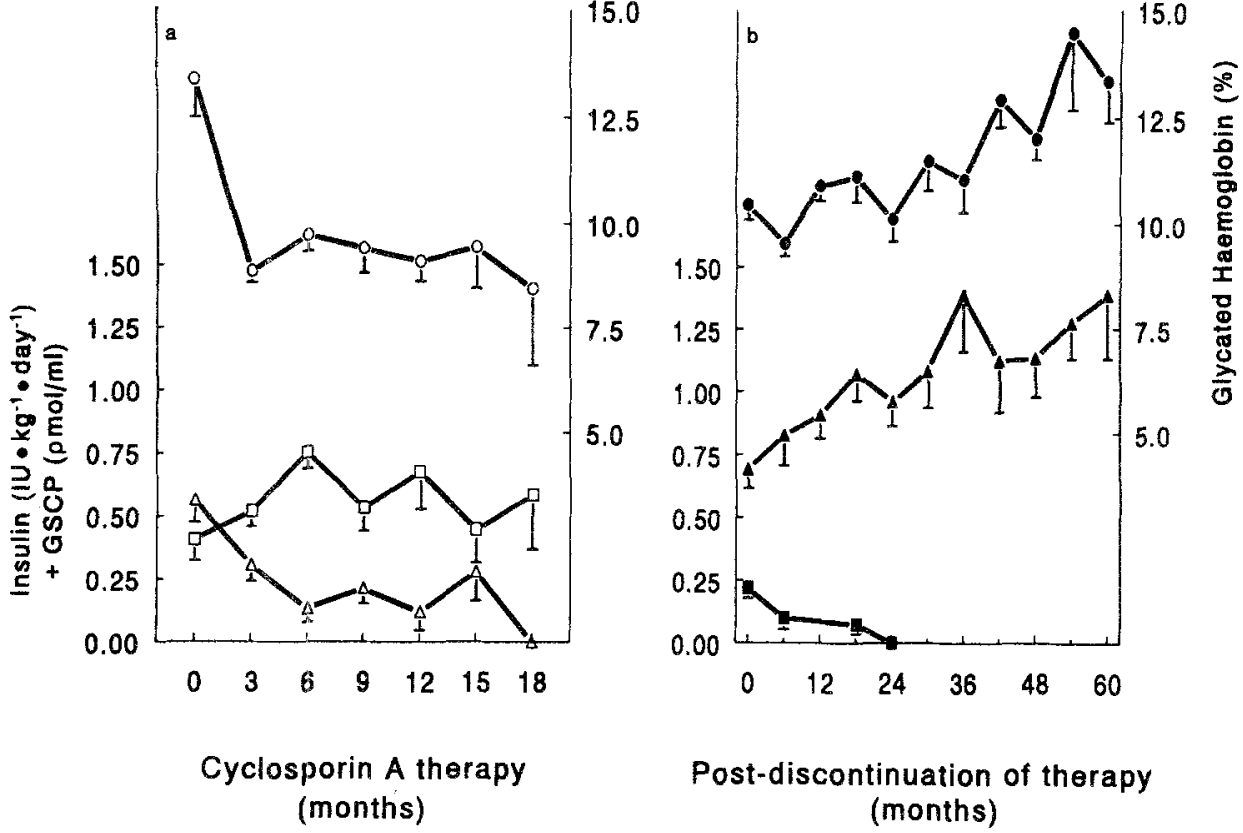

Fig. 3a,b. Metabolic effects of cyclosporin A (CsA) therapy. The mean ( \pm SEM) for glycated haemoglobin (circles), daily insulin dose (triangles) and glucagon-stimulated connecting peptide (squares) are shown postentry into study while on CsA therapy (a) and following discontinuation of CsA therapy (b). The number of children included at each time point were similar and are as follows: $13,13,10,9$, $5,5,2$, at $0,3,6,9,12,15$ and 18 months post-entry while on CsA and $12,11,10,9,8,10,9,8,6$ and 8 at 0 , $6,12,18,24,30,36,42,48,54$ and 60 months respectively after $\mathrm{CsA}$ was discontinued. Glucagon-stimulated connecting peptide (GSCP) levels were measured only until the values fell below the sensitivity of the assay quiring remissions in some of these patients [7]. A number of additional studies have confirmed the efficacy of CsA in inducing non-insulin requiring remissions $[1-5,7,9-11]$. Two randomized control trials reported $30 \%-40 \%$ of patients remained in non-insulin requiring remission at 9 months and 1 year of therapy, respectively $[2,3]$. Additionally, endogenous insulin secretion was preserved and GSCP levels were near-normal [1]. Patients most likely to enter a non-insulin requiring remission were those who had symptoms for less than 6 weeks, less than 3 weeks of insulin therapy, or both. Bougnères et al. [2] reported that CsA therapy in children between the ages of 7 and 15 years of age, was associated with a non-insulin requiring remission in approximately $50 \%$ of patients for up to 1 year of treatment. Results from the Canadian Open Study of CsA therapy in newly-diagnosed Type 1 diabetes suggested that the possibility for remission is greater in older patients [10].

Given the encouraging results in older patients this study was initiated to examine the potential benefit of CsA therapy in children with newly-diagnosed diabetes. The results of this study indicate that CsA therapy does have an effect in inducing remissions in some young children. Four of 14 (29\%) patients were able to achieve noninsulin requiring remissions including three with remissions ranging from 1 to 2.5 years. Five other patients $(36 \%)$ were able to reduce their insulin requirements to less than $0.15 \mathrm{IU} \cdot \mathrm{kg}^{-1} \cdot \mathrm{day}^{-1}$ for more than 3 months. Given the small number of patients it is not possible to assess which, if any, factors determined the response to CsA. In general however, they were older with a short duration between diagnosis and initiation of CsA (for three patients, less than 2 weeks). There were no differences in the initial GSCP or glycaemic control. As noted above, this is similar to the findings of the Canadian European Randomized Trial [2] which reported that a non-insulin requiring remission was more likely in short-duration diabetes. Side effects from CsA, in particular renal toxicity, frequently necessitated a decrease in the CsA dose.
This was associated with an increase in insulin requirements in the majority of these patients. The small number of children in this study who were able to discontinue insulin may suggest that Type 1 diabetes is more advanced or more aggressive by the time the diagnosis is made in this younger group of patients. Endogenous insulin secretory activity as measured by GSCP was maintained on CsA even though patients in remission eventually relapsed. This is supported by the results of Bougnères et al. [12] who found that children 7 to 15 years of age in remission eventually relapsed while being treated with CSA despite maintained GSCP levels. They speculated that the reduced Beta-cell mass at diagnosis may have been incapable of maintaining normal insulin sensitivity. Similar evidence has been reported recently by Finegood et al. [13]. Thus, CsA therapy may prevent additional Beta-cell loss but the residual mass may be too little by the time of diagnosis and initiation of CsA therapy.

Side effects from CsA therapy have been consistent through various studies to date and include hypertrichosis, gum hyperplasia, normocytic, normochronic anaemia, headache, tremor, peripheral paraesthesias and gastrointestinal upset. We observed similar side effects in this study. In addition, anorexia was a problem in several patients and required careful monitoring of dietary intake, to avoid significant weight loss or lack of weight gain. More serious side effects from CsA include serious infection, lymphoproliferative disease and nephrotoxicity. In all studies of CsA therapy in Type 1 diabetes there have been no reported life-threatening infections $[14,15]$. Similarly in this study there appeared to be no increase in rates of infection, however, in three children otitis media appeared to be more difficult to control and eventually resulted in discontinuation of CsA therapy. There have been no reports to date of lymphoproliferative disease in patients undergoing CsA therapy for diabetes. Epstein-Barr virus is associated with increased risk of lymphoproliferative disease in immunosuppressed patients [16-18]. Therefore, monitoring of Epstein-Barr virus infection is 
essential in treating individuals with CsA although no patients in this study became infected while on CsA therapy. Finally, nephrotoxicity is a frequent complication of CsA therapy. It has been noted in all studies to date and appears to be dose-dependent $[1,2,9]$. Histological evidence of dose-dependent CsA toxicity has been reported in patients with Type 1 diabetes and CsA therapy [6]. In doses less than $5 \mathrm{mg} \cdot \mathrm{kg}^{-1} \cdot$ day $^{-1}$, with whole blood trough levels less than $300 \mathrm{ng} / \mathrm{ml}$ in adults or $600 \mathrm{ng} / \mathrm{ml}$ in children or both, there does not appear to be any histological renal toxicity. In this study calculated creatinine clearance declined while on CsA therapy. Following discontinuation of CsA creatinine clearance increased and returned to normal values. A decline in creatinine clearance of greater than $30 \%$ prompted a decrease in CsA therapy which frequently resulted in a decline in endogenous insulin secretion in addition to an increase in insulin requirements. More sensitive indicators of renal toxicity may have demonstrated minor changes in renal function. However, as discussed above the blood levels of CsA in these patients have not been associated with histological renal toxicity [6]. In the eight patients still being followed-up in our clinic all but one have microalbuminuria less than $30 \mu \mathrm{g} / \mathrm{min}$ more than 6 years after discontinuing CsA. The other patient is a 16-year-old male who had a non-insulin requiring remission for 15 months. Eighty-nine months after discontinuing CsA he had microalbuminuria of $52.3 \mu \mathrm{g} / \mathrm{min}$. It is difficult to determine whether this is due to CsA nephrotoxicity, diabetes or both.

All side effects from CsA therapy disappeared following its discontinuation. For the period of post-CsA observation, which is now 5 years for all but one patient, there have been no significant side effects. Growth and renal function have been normal, and normal growth has also been reported in children who have received a renal transplant and are on CsA therapy $[19,20]$.

In summary, CsA had limited efficacy in modifying the initial course of Type 1 diabetes in this very young age group. Although several side effects of CsA were noted all of these were reversible following discontinuation of CsA and there have been no long-term effects.

The efficacy of CsA in newly-diagnosed patients with Type 1 diabetes suggest a role for immunomodulation once a safe therapy is available. However, the results of this study indicate that treating very young children at the time of diagnosis may be too late for most to achieve a non-insulin requiring remission. Future intervention studies should be directed at treating the pre-clinical state such as that recently reported by Elliott and Chase [21], using nicotinamide.

Acknowledgements. We gratefully acknowledge Dr. G. Murphy and Sandoz Canada for supplying cyclosporin A. We also thank Ms. H. Sim and Mr. G. Ryan for data analysis and Ms. E. Allison for the preparation of this manuscript.

\section{References}

1. Feutren G, Papoz L, Assan R, Vialettes et al. (1986) Cyclosporin increases the rate and length of remission in insulin-dependent diabetes of recent onset. Lancet II: 119-124
2. Canadian-European randomized control trial group (1988) Cyclosporine-induced remission of IDDM after early intervention. Association of one year of cyclosporine with enhanced insulin secretion. Diabetes 37: 1574-1582

3. Stiller CR, Dupré J, Gent Met al. (1987) Effects of cyclosporine in recent-onset juvenile Type 1 diabetes: impact of age and duration of disease. J Pediatr 111: 1069-1072

4. Bougneres PF, Carel JC, Castano L et al. (1988) Factors determining early remission of Type 1 diabetes in children treated with cyclosporin A. N Engl J Med 38: 663-670

5. Bach JF (1987) Cyclosporine in insulin-dependent diabetes mellitus. J Pediatr 111: 1073-1074

6. Feutren G (1988) Functional consequences and risk factors of chronic cyclosporin A nephrotoxicity in Type 1 diabetes trials. Transplant Proc 20: 356-366

7. Stiller CR, Dupré J, Gent M et al. (1984) Effects of cyclosporine immunosuppression in insulin-dependent diabetes mellitus of recent onset. Science 223: 1362-1367

8. Laupacis A, Stiller CR, Gardell C et al. (1983) Cyclosporin prevents diabetes in $B B$ Wistar rats. Lancet I: $10-12$

9. Assan R, Sachs MD, Laborie C et al. (1985) Metabolic and immunologic effect of cyclosporine in recently diagnosed Type 1 diabetes mellitus. Lancet I: 67-71

10. Dupré J, Stiller CR, Gent Met al. (1988) Effects of immunosuppression with cyclosporine in insulin dependent diabetes mellitus of recent onset: the Canadian open study of 44 months. Transplant Proc 20: 184-192

11. Stiller CR, Keown PA (1984) Cyclosporine therapy in perspective: In Morris PJ, Tilney NL (eds) Progress in transplantation. Churchill Livingstone, Edinburgh, pp 11-45

12. Bougnères PF, Landais $P$, Boisson $C$ et al. (1990) Limited duration of remission of insulin dependency in children with recent overt type 1 diabetes treated with low-dose cyclosporin. Diabetes 39: 1264-1272

13. Finegood D, Hramiak IM, Dupré J (1990) Relationship between stimulated C-peptide responses and insulin sensitivity in insulindependent diabetics treated with cyclosporin. Diabetologia 33 [Suppl]: A208 (Abstract)

14. Bach JF (1988) The risk/benefit ratio in immunointervention for autoimmune diseases. J Autoimmun 1: 711-720

15. Bach JF (1992) Immunointervention in autoimmune diseases from cellular selectivity to autoantigen specificity. $\mathbf{J}$ Autoimmun 5 [Suppl]: A 3-10

16. Editorial (1984) Lymphoma in organ transplant recipients. Lancet I: 601-603

17. Hanto DW, Frizzera G, Gajl-Peczalska KJ, Simmons RL (1985) Epstein-Barr virus, immunodeficiency and B cell lymphoproliferation. Transplantation 39: 461-472

18. Ho M, Jaffe R, Miller G et al. (1988) The frequency of EpsteinBarr virus infection and associated lymphoproliferative syndrome after transplantation and its manifestations in children. Transplantation 45: 719-727

19. Ettenger RB, Rosenthal JT, Marik J et al. (1991) Long-term results with cyclosporine immune suppression in pediatric cadaver renal transplantation. Transplant Proc 23: 1011-1012

20. Klore B, Strom TM, Hahn H et al. (1991) Remarkable long-term prognosis and excellent growth in kidney-transplant children under cyclosporine monotherapy. Transplant Proc 23: 1013-1017

21. Elliott RB, Chase HP (1991) Prevention of delay of Type 1 (insulin dependent) diabetes mellitus in children using nicotinamide. Diabetologia 34: 362-365

Received: 19 March 1992

and in revised form: 25 May 1992

Dr.P.R.Atkison

University Hospital

339 Windermere Road

London, Ontario N6A 5 A5

Canada 